\title{
The effects of therapy on spasticity utilizing a motorized exercise-cycle
}

\author{
J Rösche ${ }^{1}$, C Paulus ${ }^{2}$, U Maisch ${ }^{1}$, A Kaspar ${ }^{2}$, E Mauch ${ }^{2}$ and HH Kornhuber ${ }^{1}$ \\ ${ }^{1}$ Department of Neurology, University of Ulm, D-89075 Ulm, Germany and ${ }^{2}$ Fachklinik für Neurologie Dietenbronn, \\ D-88477 Schwendi, Germany
}

\begin{abstract}
F-wave amplitudes have been used to demonstrate changes of motor neuron excitability in patients receiving pharmacological antispastic therapy as well as in those having physiotherapy. In this study it is shown that F-wave amplitudes can also be used to document changes of motor neuron excitability as an effect of the therapy with a motorized exercise-cycle, which moves the legs of paraplegic patients in a way similar to cycling. Ten Fwaves were recorded immediately before and after the therapy with a motorized exercise-cycle in 70 legs of 35 patients with spastic paraparesis. Mean F-wave amplitude, mean F-wave/Mresponse ratio and maximum $\mathrm{F}$-wave/M-response ratio were significantly lower after therapy than before. Conclusion: The antispastic effect of the therapy with a motorized exercise-cycle may be documented by a decrease of F-wave-amplitude parameters.
\end{abstract}

Keywords: spastic paraplegia; physical therapy; exercise-cycle; electrophysiology; F-waves

\section{Introduction}

F-wave size has been shown to reflect changes of motor neuron excitability. ${ }^{1}$ Several authors found that it is increased in those with spasticity. ${ }^{2-8}$ But it is also somewhat increased in healthy subjects when obtained after stimulation with frequencies above $0.2 \mathrm{~Hz}^{4}$ Therefore F-wave studies to evaluate antispastic therapeutic effects should be performed at a stimulation rate not higher than $0.2 \mathrm{~Hz}$.

The F-wave amplitude changed parallel to the muscle tone after an acute cerebral insult ${ }^{3}$ and was reduced by several medicines in long standing poststroke hemiparesis. ${ }^{5,9}$ Intrathecal Baclofen reduced the mean F-wave amplitude even about $60 \% .{ }^{10}$ But physiotherapy was also shown to reduce the F-waveamplitude. ${ }^{11}$ In spastic hemiparesis following a stroke T-, H-reflex-amplitude ratios as well as F-wave amplitude ratios were increased. But only the F-wave amplitude ratios decreased after treatment. ${ }^{5}$ The Fwave/M-response ratio was also shown to correlate with the increase of muscle tone in patients with multiple sclerosis. ${ }^{12}$ It was more sensitive to changes of lower motoneuron excitability induced by physiotherapy than the F-wave-amplitude itself. ${ }^{11}$

Physiotherapy plays an important part in the rehabilitation of patients with spasticity. But since qualified physiotherapy is not available every day for patients living at home, therapy with a motorized exercise-cycle is often necessary to treat spasticity in

Correspondence: Dr med J Rösche people with multiple sclerosis. ${ }^{13}$ The aim of this work is to investigate whether the effects of antispastic treatment with a motorized exercise-cycle can be documented by $\mathrm{F}$-wave-amplitude parameters.

\section{Materials and methods}

We examined 35 patients with spastic paraparesis; 31 had multiple sclerosis, one had multiple system atrophy and two patients had familial spastic paraplegia. In the remaining patient a spinal tumor was detected. Their age ranged from 32 to 67 years (Mean 49; SD 10.32). Duration of disease ranged from 7 to 39 years (Mean 15.92; SD 9.12). All had increased muscle tone, decreased muscle force and positive Babinski signs.

Electrophysiological examination was performed with the 'Toennies Multiliner' immediately before and after a treatment with a motorized exercisecycle. Electrophysiological examination and treatment were performed in the same room. The patients were tested in the supine position and were encouraged to relax as much as possible. M-response was recorded after surface electrode supramaximal electrostimulation of the tibial nerves at their distal points from the flexor hallucis brevis muscles. Then $10 \mathrm{~F}$-waves were recorded after supramaximal electrostimulation at a rate of $0.2 \mathrm{~s}$. The whole procedure was performed on each leg before and after treatment. The shortest Fwave-latency, M-response-amplitude and F-waveamplitudes were registered for evaluation. All parameters before and after treatment were compared with the Wilcoxon paired test. 
Physical therapy was performed using MOTOmed ${ }^{\mathrm{R}}$, which is a motorized exercise-cycle for the reduction of spasticity by rhythmical movement of the legs. In this study treatment was performed for $30 \mathrm{~min}$ with a speed of 40 rounds per minute.

\section{Results}

The amplitude of the M-response was $17.3 \mathrm{mV}$ (SD 8.16) before and $17.44 \mathrm{mV}$ (SD 8.07) after the treatment with a motorized exercise-cycle. In 38 legs the amplitude of the M-response increased ( $\mathrm{n}$ sign). The shortest F-wave-latencies were $50.2 \mathrm{msec}$ (SD 3.73) before and $50.5 \mathrm{msec}$ (SD 3.95) after the treatment with the motorized exercise-cycle. In 36 legs the latencies increased (n sign). Thus there was neither a significant change in F-wave-latencies nor in Mresponse-amplitudes. The mean of F-wave-amplitudes was $0.62 \mathrm{mV}$ (SD 0.41; SEM 0.049) before and $0.56 \mathrm{mV}$ (SD 0.35; SEM 0.041) after treatment. In 42 legs the mean of F-wave-amplitudes decreased $(P<0.05)$. The maximum of $\mathrm{F}$-wave amplitudes was $0.95 \mathrm{mV}$ (SD 0.53; SEM 0.063) before and $0.86 \mathrm{mV}$ (SD 0.46; SEM 0.055) after treatment. In 44 legs the maximum of F-wave-amplitudes decreased, but the decrease failed to reach significance $(0.05<P<0.1)$. Thus there was a slight significant decrease of mean amplitudes after treatment with the motorized exercisecycle when only the F-waves were considered. But the decrease was clearly significant when the relation of Fwaves to the M-responses was taken into account (Figure 1). The Mean-F-wave/M-response ratio was $4.23 \%$ (SD 3.06; SEM 0.37) before and 3.72\% (SD 2.38, SEM 0.28) after treatment.

The mean-F-wave/M-response ratio decreased in 47 legs $(P<0.001)$. The maximum-F-wave/M-response ratio was $6.71 \%$ (SD 5.3; SEM 0.63) before, and 5.89\%: (SD 4.24; SEM 0.51) after treatment. Maximum-F-wave/M-response ratio decreased in 45 legs $(P<0.02)$. Thus both $\mathrm{F}$-wave-M-response ratios were lower after but not before the treatment with the motorized exercise-cycle. In 16 legs none of the four Fwave-amplitude parameters taken into consideration decreased. But in only two patients was there no decrease of F-wave-amplitude parameters in both legs.

\section{Discussion}

Normal values for F-wave amplitudes were published by Fisher et al. ${ }^{14}$ They found a mean value for the mean F-wave/M-response ratio of 2.2\% (SD 0.7; range $1.2-3.5 \%$ ). In comparison to their values the mean Fwave/M-response ratio in our patients was clearly increased before and still somewhat subsequent to treatment with a motorized exercise-cycle.

Milanov $^{6}$ hypothesized that alpha-neuron-excitability, as measured by F-wave-amplitudes, usually

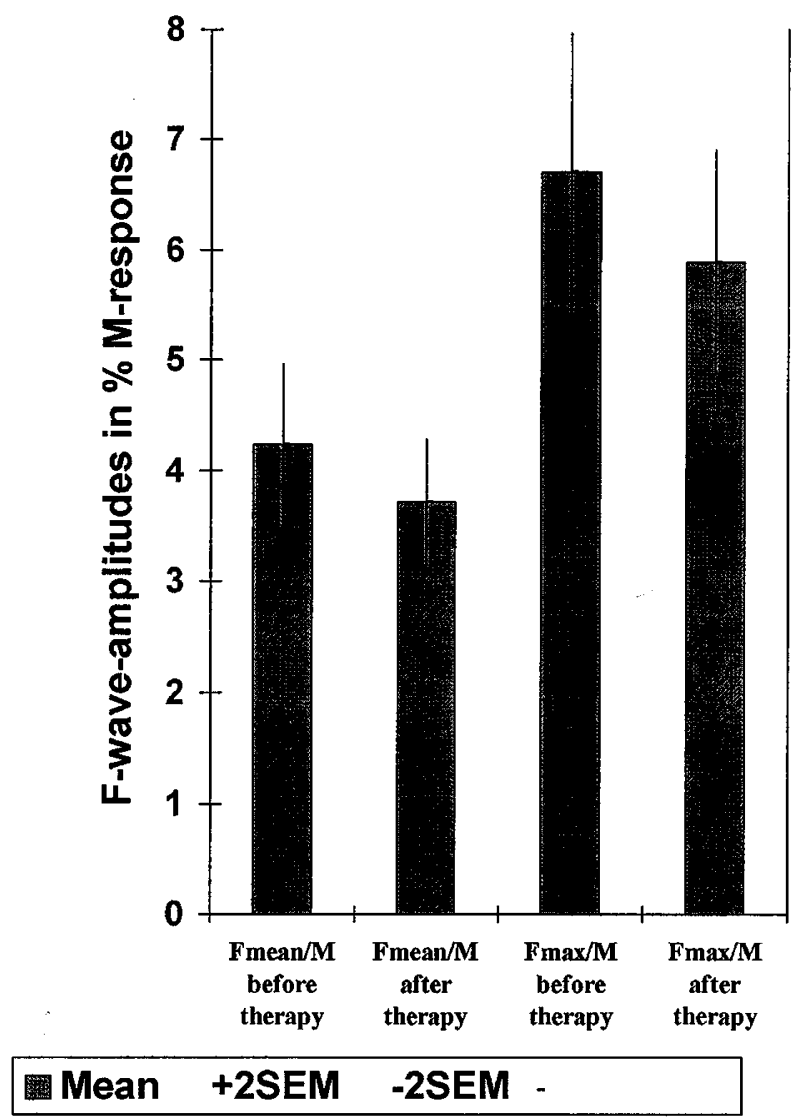

Figure 1 Changes of F-wave/M-response ratio after $30 \mathrm{~min}$ treatment with a motorized exercise-cycle

develops secondarily after alteration of some other segmental mechanism in those with spasticity (eg: increased gamma-motoneurone activity, altered interneurone activity, decreased presynaptic inhibition). Therefore several different mechanisms may have led to the high F-wave-amplitudes observed in this study. Not all of them may have been influenced by the treatment with a motorized exercise-cycle. In addition secondary changes in the muscles may occur. This may be the reason for the decrease of F-wave-amplitude/Mresponse ratio after the treatment with a motorized exercise-cycle not being observed in every treated leg. Since F-wave/M-response ratios proved to be more significant to changes of motoneuron excitability than were the raw F-wave-amplitudes, it is clear that an effect on motoneuron-excitability is sometimes only noticed by comparing $\mathrm{F}$-wave/M-response ratios. The antispastic effect of treatment with a motorized exercise-cycle can be documented by recording Fwave-amplitudes. 


\section{References}

1 Fox JE, Hitchcock ER. F wave size as a monitor of motorneuron excitability: the effect of deafferentation. J Neurol Neurosurg Psychiat 1987; 50: $453-459$.

2 Eisen A, Odusote K. Amplitude of the F wave: A potential means of documenting spacticity. Neurology 1979; 29: 1306-1309.

3 Fierro B, Raimondo D, Modica A. Analysis of F response in upper motoneurone lesions. Acta Neurol Scand 1990; 82: $329-$ 334.

4 Fierro B, Raimondo D, Modica A. F-wave study at different stimulation rates in upper motoneurone lesions. Electromyogr clin Neurophysiol 1993; 33: $27-31$.

5 Milanov GI. A comparison of methods to assess the excitability of lower motorneurones. Can J Neurol Sci 1992; 19: 64-68.

6 Milanov I. Examination of the segmental pathophysiological mechanisms of spasticity. Electromyogr clin Neurophysiol 1994; 34: $73-79$.

7 Schiller HH, St ̊̊lberg E. F responses studied with single fibre EMG in normal subjects and spastic patients. J Neurol Neurosurg Psychiat 1978; 41: 45-53.

8 Smith SJM et al. F responses and central motor conduction in multiple sclerosis. Electroencephalogr Clin Neurophysiol 1989; 74: $438-443$.

9 Milanov I, Georgiev D. Mechanisms of tizanidine action on spasticity. Acta Neurol Scand 1994; 89: 274-279.

10 Dressnandt J, Auer C, Conrad B. Influence of Baclofen upon the Alpha-Motoneuron in Spasticity by Means of F-Wave Analysis. Muscle \& Nerve 1995; 18: $103-107$.

11 Rösche $\mathbf{J}$ et al. Effects of physiotherapy on F-wave-amplitudes in spasticity. Electromyogr clin Neurophysiol 1996; 36: in press.

12 Rösche J, Rüb K, Mauch E, Kornhuber HH. Das Amplitudenverhältnis F-Welle/M-Antwort korreliert mit der Spastik bei multipler Sklerose. Nervenheilkunde 1994; 13: S6-S8.

13 Kornhuber HH. Forschung mit dem Ziel, den Patienten direkt zu helfen: Symptomatische Therapie der Multiplen Sklerose als Grundbehandlung und als Voraussetzung für wirksamere Immunosupression. In: Hertie-Stiftung (ed). Multiple Sklerose, Hertie, Frankfurt/M 1987, pp. 83-95.

14 Fisher MA, Hoffen B, Hultman Ch. Normative F wave values and the number of recorded F waves. Muscle Nerve 1994; 17: $1185-1189$ 